\title{
Global existence for the critical dissipative surface quasi-geostrophic equation
}

\author{
Omar Lazar *
}

\begin{abstract}
In this article, we study the critical dissipative surface quasi-geostrophic equation (SQG) in $\mathbb{R}^{2}$. Motivated by the study of the homogeneous statistical solutions of this equation, we show that for any large initial data $\theta_{0}$ liying in the space $\Lambda^{s}\left(H_{u l o c}^{s}\left(\mathbb{R}^{2}\right)\right) \cap L^{\infty}\left(\mathbb{R}^{2}\right)$ the critical (SQG) has a global weak solution in time for $1 / 2<s<1$. Our proof is based on an energy inequality verified the $(S Q G)_{R, \epsilon}$ equation which is nothing but the equation (SQG) equation with truncated and regularized initial data. By classical compactness arguments, we show that we are able to pass to the limit $(R \rightarrow \infty, \epsilon \rightarrow 0)$ in $(S Q G)_{R, \epsilon}$ and that the limit solution has the desired regularity.
\end{abstract}

Keywords: Quasi-geostrophic equation, fluid mechanics, Riesz transforms.

\section{Introduction}

We consider the initial value problem of the $2 \mathrm{D}$ dissipative surface quasi-geostrophic equation :

$$
\left\{\begin{array}{l}
\partial_{t} \theta(x, t)+u . \nabla \theta+\Lambda^{\alpha} \theta=0 \\
u(\theta)=\mathcal{R}^{\perp} \theta \\
\theta(0, x)=\theta_{0}(x)
\end{array}:(S Q G)_{\alpha}\right.
$$

where

$$
\Lambda^{\alpha} \theta=(-\Delta)^{\alpha / 2} \theta=C_{\alpha} P . V . \int_{\mathbb{R}^{n}} \frac{\theta(x)-\theta(x-y)}{|y|^{2+\alpha}} d y,
$$

$C_{\alpha}$ is a positive constant and $\theta: \mathbb{R}_{+} \times \mathbb{R}^{2} \rightarrow \mathbb{R}$ is a scalar function. Note that when $0<\alpha<1$ and $\theta$ regular enough we can get rid of the principal value since the integral is then absolutely convergent. Here $\alpha \in(0,2]$ is a fixed parameter and the velocity $u=\left(u_{1}, u_{2}\right)$ is divergence free and determined by the Riesz transforms of the potential temperature $\theta$ via the formula:

$$
u=\left(-\mathcal{R}_{2} \theta, \mathcal{R}_{1} \theta\right)=\left(-\partial_{x_{2}}(-\Delta)^{-1 / 2} \theta, \partial_{x_{1}}(-\Delta)^{-1 / 2} \theta\right) .
$$

We can distinguish 3 cases depending on the value of $\alpha$. Namely, when $\alpha>1, \alpha=1, \alpha<1$ which are called respectively the sub-critical, critical and super-critical cases. Actually, the more physically relevant case is the critical one due to the term $(-\Delta)^{1 / 2}$ which models the Ekman pumping; as such it provides understanding of the quasi geostrophic flows. Moreover, some authors (see e.g. [8]) have noticed that there is a similarity between the critical case and the 3D Navier-Stokes equation. In this paper, we will focus only on the critical case $(\alpha=1)$ which we will denote by $(S Q G)$. The existence of weak solutions for this equation was proved by Resnick in [18] when the initial data lies in $L^{2}\left(\mathbb{R}^{2}\right)$. Many authors have studied the critical case, among them we can cite the work by Constantin, Córdoba and $\mathrm{Wu}$ [7] in which they showed that there is a unique global solution when $\theta_{0} \in H^{1}$ and under a smallness assumption on $\left\|\theta_{0}\right\|_{L^{\infty}}$. In [4], Caffarelli and Vasseur proved the global regularity of weak solutions with $L^{2}$ data. Their proof is based on De Giorgi techniques. Another important result is the one of Kiselev, Nazarov and Volberg [13] wherein the authors used a non local maximum principle verified by the modulus of continuity at time 0 . They

\footnotetext{
*Université Paris-Est, LAMA (UMR 8050), UPEMLV, UPEC, CNRS, F-77454, Marne-la-Vallée, France. Email: omar.lazar@univ-mlv.fr
} 
showed that all smooth periodic initial data gives rise to a unique global smooth solution. Abidi and Hmidi [1] proved the global well-posedness of the critical dissipative quasi-geostrophic equation for large initial data belonging to the critical Besov space $\dot{B}_{\infty, 1}^{0}\left(\mathbb{R}^{2}\right)$. In [14], Marchand showed the existence and regularity of global weak solutions to the quasi-geostrophic equations when the initial data belongs to $L^{p}(p>4 / 3)$ or to $\dot{H}^{-1 / 2}$.

Whereas the sub critical case $(\alpha>1)$ is globally well understood (see e.g [9]), the global regularity issue for large data in the super critical case $(\alpha<1)$ is still open. Nevertheless, in the super critical case, several results have been obtained, for instance, we can cite the work by Chae and Lee [6] where the authors showed the global regularity for small initial data in spaces $B_{2,1}^{2-2 s}$. Global results for small initial data in Sobolev spaces $H^{m}$ with $m<2$ can also be found in [10] and [12].

In this paper, we show the existence of a global weak solution of $(S Q G)$ for an initial data $\theta_{0} \in \Lambda^{s}\left(H_{\text {uloc }}^{s}\left(\mathbb{R}^{2}\right)\right) \cap L^{\infty}\left(\mathbb{R}^{2}\right)$. This space is a well adapted space when we plan to construct space-time homogeneous statistical solutions in the sense of Vishik and Fursikhov ([19], [20]) for the $(S Q G)$ equation. Roughly speaking, those statistical solutions have necessarily infinite energy, that is why the classical case $\theta_{0} \in L^{2}$ is not allowed. Unlike the Navier-Stokes equation case, we cannot consider the case $\theta_{0} \in L_{u l o c}^{2}$ as in the pioneer work by Lemarié-Rieusset [16], [17], and more recently Basson [2]. This is due to the fact that Riesz transforms are not well-defined in that space. Actually, to overcome this difficulty, we need more decay at infinity for the kernel of the Riesz transforms operator, so if we give more oscillations to $\theta_{0}$ we will be able to consider the case $\theta_{0} \in L_{u l o c}^{2}$. As a matter of fact, if we put $\theta_{0}=\Lambda^{s} w_{0} \in \Lambda^{s}\left(H_{u l o c}^{s}\left(\mathbb{R}^{2}\right)\right)$ with $1 / 2<s<1$, then we can show that the $(S Q G)$ equation makes sense in $\mathcal{D}^{\prime}\left(\mathbb{R}^{2}\right)$. Since we plan to use the maximum principle we futher suppose that $\theta_{0} \in L^{\infty}$.

Our main result is the following theorem :

Theorem 1. Assume that $1 / 2<s<1$, and $\theta_{0} \in \Lambda^{s}\left(H_{u l o c}^{s}\right) \cap L^{\infty}$, then the (SQG) equation has at least one global weak solution. Moreover for any $T<\infty$, the solution belongs to the following spaces:

$$
\theta \in L^{\infty}\left([0, T], L_{\text {uloc }}^{2}\right) \cap\left(L_{t}^{2}\left([0, T], \dot{H}^{1 / 2}\right)\right)_{u l o c}
$$

and

$$
w \in L^{\infty}\left([0, T], H_{u l o c}^{s}\right) \cap\left(L_{t}^{2}\left([0, T], \dot{H}^{s+1 / 2}\right)\right)_{u l o c} .
$$

Futhermore, the following inequality holds for all $t \leq T$

$$
\|w(x, t)\|_{L_{t}^{\infty} H_{u l o c}^{s}\left(\mathbb{R}^{2}\right)}^{2} \leq c e^{C T},
$$

where $c$ and $C$ are two constants depending only on $\left\|\theta_{0}\right\|_{L^{\infty}\left(\mathbb{R}^{2}\right)}$ and $\left\|w_{0}\right\|_{H_{u l o c}^{s}\left(\mathbb{R}^{2}\right)}$.

Remark 2. Beside giving an extension of Resnick's theorem (see theorem 3.1), this theorem would also allow us to show the existence of statistical solutions in the sense of Vishik and Fursikhov [19], [20].

This paper is organized as follows. In the next section we give a definition of the spaces and fix some notations. In the third section, we review some well known results we will use in the proof of our main theorem and we prove an energy inequality verified by the truncated equation associated with a regularized and truncated initial data. In the fourth section, we show that we can pass to the weak limit with respect to the parameters, this allows us to conclude the proof of our main result.

\section{Preliminaries and notations}

In this section, we recall some definitions and well-known results that we will use in the proof of our main theorem. 
We start with the definition of the space $L_{u l o c}^{p}\left(\mathbb{R}^{2}\right)$. This space belongs to the more general class of the so-called non homogenous Morrey-Campanato spaces defined by:

$$
M_{q}^{p}\left(\mathbb{R}^{2}\right)=\left\{f \in L_{l o c}^{p}\left(\mathbb{R}^{2}\right) \mid \sup _{x \in \mathbb{R}^{2}} \sup _{0<R<1} R^{\frac{2}{p}-\frac{2}{q}}\|f\|_{L^{q}(B(x, R))}<\infty\right\} .
$$

For $p=q$ we get the $L_{u l o c}^{p}\left(\mathbb{R}^{2}\right)$ space. In the sequel, we will use the following definitions of the $L_{\text {uloc }}^{p}\left(\mathbb{R}^{2}\right)$ spaces :

Definition 3. Let us fix a positive test function $\phi_{0}$ such that $\phi_{0} \in \mathcal{D}\left(\mathbb{R}^{2}\right)$ and

$$
\begin{cases}\phi_{0}(x)=1 & \text { if }|x| \leq 2 \\ \phi_{0}(x)=0 & \text { if }|x| \geq 3\end{cases}
$$

We define the set of all translations of the function $\phi_{0}$ as $B_{\phi_{0}}=\left\{\phi_{0}(x-k), k \in \mathbb{Z}^{2}\right\}$. In the following, this set will be denoted by $B$ or $B_{\phi_{0}}$.

Remark 4. Let $1 \leq p \leq \infty, f \in L_{\text {uloc }}^{p}\left(\mathbb{R}^{2}\right)$ if and only if $f \in L_{\text {loc }}^{p}\left(\mathbb{R}^{2}\right)$ and the following norm is finite

$$
\|f\|_{L_{u l o c}^{p}\left(\mathbb{R}^{2}\right)}=\sup _{\phi \in B_{\phi_{0}}}\|\phi f\|_{L^{p}\left(\mathbb{R}^{2}\right)} .
$$

We will also use the following useful equivalent norms:

$$
\|f\|_{L_{u l o c}^{p}\left(\mathbb{R}^{2}\right)}^{p} \approx \sup _{k \in \mathbb{Z}^{2}} \int_{k+[0,1]^{2}}|f(x)|^{p} d x \approx \sup _{k \in \mathbb{Z}^{2}}\|\phi(x-k) f\|_{L^{p}\left(\mathbb{R}^{2}\right)}
$$

Let us also recall the following useful lemma:

Lemma 5. Let $f \in L^{1}\left(\mathbb{R}^{2}\right)$ and $g \in L_{\text {uloc }}^{p}\left(\mathbb{R}^{2}\right)$ then the convolution product is well defined (a.e.) and we have $\|f * g\|_{L_{u l o c}^{p}\left(\mathbb{R}^{2}\right)} \leq\|f\|_{L^{1}}\|g\|_{L_{u l o c}^{p}}$.

Proof. As mentioned in [2], if we put $K=x_{0}+[0,1]^{2}$, it suffices to write:

$$
\left\|\int|f(y) g(x-y)| d y\right\|_{L^{p}(K)} \leq \int|f(y)|\left\|g(x-y) \mathbb{1}_{x \in K}\right\|_{L_{x}^{p}} d y \leq\|f\|_{L^{1}}\|g\|_{L_{u l o c}^{p}}
$$

Throughout this paper, we will make use of a test function $\psi$ constructed as follows. We introduce a positive test function $\psi_{0}$ supported in $[-4,4]^{2}$ such that $\psi_{0} \geq 0$ and

$$
\begin{cases}\phi_{0}(x)=1 & \text { if }|x| \leq 3 \\ \phi_{0}(x)=0 & \text { if }|x| \geq 4\end{cases}
$$

Now, we construct the space of all translations of $\psi_{0}$ that is:

$$
B_{\psi_{0}}=\left\{\psi_{0}\left(x-x_{0}\right), x_{0} \in \mathbb{Z}^{2}\right\} .
$$

Then, as we did before, we define $\psi(x)=\psi_{0}(x-k)$ where $k \in \mathbb{Z}^{2}$. Note that by construction, the function $\psi$ is equal to 1 in a neighborhood of the support of $\phi$.

We will also denote $A \lesssim B$ if $A$ is less than $B$ up to a positive multiplicative constant which can be different from line to line, and the positive constant $C$ which appears in some estimations can be different as well. Keep in mind that those constants may depend on certain controlled norms of the initial data.

We recall the definition of the Sobolev space $H^{s}\left(\mathbb{R}^{2}\right)$ where $s \in \mathbb{R}$ :

Definition 6. A distribution $f \in \mathcal{S}^{\prime}\left(\mathbb{R}^{2}\right)$ is in $H^{s}\left(\mathbb{R}^{2}\right)$ if $\hat{f}$ is locally integrable on $\mathbb{R}^{2}$ and

$$
\|f\|_{H^{s}} \equiv\left(\int_{\mathbb{R}^{2}}\left(1+|\xi|^{2}\right)^{s}|\hat{f}(\xi)|^{2} d \xi\right)^{1 / 2}<\infty .
$$


The Sobolev spaces can be defined through the fractional Laplacian, and more precisely we have the following lemma.

Lemma 7. If $0<s<1$ then the norm :

$$
N(f)=C_{s}\left(\int|f(x)|^{2} d x+\iint \frac{|f(x)-f(y)|^{2}}{|x-y|^{n+2 s}} d x d y\right)^{1 / 2}
$$

is finite on $H^{s}$ and this norm is equivalent to the usual $H^{s}$ norm.

Proof. See e.g. Prop 1.37 p 28, [3]

Now, let us recall the definition of the $H_{\text {uloc }}^{s}\left(\mathbb{R}^{2}\right)$ space, where $s \in \mathbb{R}$ :

Definition 8. Let $\phi_{0}$ be a positive test function such that $\phi_{0} \in \mathcal{D}\left(\mathbb{R}^{2}\right)$ such that $\phi(x)=1$ if $x \in$ $[-1,1]^{2}$ and let $B=\left\{\phi_{0}(x-k), k \in \mathbb{Z}^{2}\right\}$. We say that $f \in H_{\text {uloc }}^{s}\left(\mathbb{R}^{2}\right)$ if and only if $f \in H_{\text {loc }}^{s}\left(\mathbb{R}^{2}\right)$ and if the following norm is finite:

$$
\|f\|_{H_{u l o c}^{s}}^{2}\left(\mathbb{R}^{2}\right)=\sup _{\phi \in B}\|\phi f\|_{H^{s}}
$$

Remark 9. We can easily see that those defintions do not depend on the choice of the test function since we take the supremum over all translations of $\phi$. Let us precisely state that $f \in \Lambda^{s}\left(\dot{H}_{\text {uloc }}^{s}\left(\mathbb{R}^{2}\right)\right)$ if there exists $g \in \dot{H}_{\text {uloc }}^{s}\left(\mathbb{R}^{2}\right)$ s.t $f=\Lambda^{s} g$. Let us also define some space-time norms that we will use throughout this paper, namely $\left(L_{T}^{\infty} \dot{H}^{s}\right)_{u l o c},\left(L_{T}^{2} \dot{H}^{s}\right)_{u l o c}$, where $s \in[0,1]$.

$$
\begin{aligned}
\|w\|_{\left(L_{T}^{2} \dot{H}^{s}\right)_{u l o c}}^{2} & =\sup _{\phi \in B} \int_{0}^{T} \int \phi\left|\Lambda^{s} w(x, s)\right|^{2} d x d s<\infty, \\
\|w\|_{\left(L_{T}^{\infty} \dot{H}^{s}\right)_{u l o c}} & =\sup _{t \in[0, T]} \sup _{\phi \in B} \int \phi\left|\Lambda^{s} w(x, t)\right|^{2} d x<\infty .
\end{aligned}
$$

In the sequel, we shall use the following notations:

$$
A_{\phi}(w)=\int\left(\frac{|w|^{2}}{2} \phi+\frac{\left|\Lambda^{s} w\right|^{2}}{2} \phi\right) d x \quad \text { and } \quad A(w)=\int\left(\frac{|w|^{2}}{2}+\frac{\left|\Lambda^{s} w\right|^{2}}{2}\right) d x
$$

The next lemma will be useful for the proof of our result :

Proposition 10. Let $0<s<1$; the following norms are equivalent on $H_{\text {uloc }}^{s}\left(\mathbb{R}^{2}\right)$ :

- $\|w\|_{a}=\sup _{\phi \in B}\left\{A_{\phi}(w)<\infty\right\}$

- $\|w\|_{b}=\sup _{\phi \in B}\{A(w \phi)<\infty\}$.

\section{Proof.}

Since $0 \leq \phi \leq 1$, we have $\frac{|w \phi|^{2}}{2} \leq \frac{|w|^{2}}{2} \phi$ and then

$$
\sup _{\phi \in B} \int \frac{|w \phi|^{2}}{2} d x \leq \sup _{\phi \in B} \int \frac{|w|^{2}}{2} \phi d x \text {. }
$$

Now, let us prove the reverse inequality. Suppose that we control $\sup _{\phi \in B} \int \frac{|w|^{2}}{2} \phi d x$. We put $\eta^{2}(x)=\phi(x)$, then

$$
\sup _{\phi \in B} \int \frac{|w|^{2}}{2} \phi d x \leq \sup _{\eta^{2} \in B_{\eta^{2}}} \int \frac{|w \eta|^{2}}{2} d x .
$$

These two previous inequalities show the following equivalence of norm on $L_{\text {uloc }}^{2}$

$$
\|w\|_{L_{\text {uloc }}^{2}}^{2} \sim \sup _{\phi \in B} \int \frac{|w|^{2}}{2} \phi d x \sim \sup _{\phi \in B} \int \frac{|w \phi|^{2}}{2} d x .
$$


Now, we just have to prove that if $\Lambda^{s}(\phi w) \in L^{2}$ then $\phi \Lambda^{s} w \in L^{2}$ and the converse. Throughout this paper, we will frequently make use of the commutator between an operator $T$ and a function $\phi$ defined by the formula

$$
[T, \phi] f=T(\phi f)-f T(\phi) .
$$

Then, for all $\phi \in B_{\phi_{0}}$ and $\psi \in B_{\psi_{0}}$ we can write

$$
\psi\left[\Lambda^{s}, \phi\right] w+(1-\psi)\left[\Lambda^{s}, \phi\right] w=\Lambda^{s}(\phi w)-\phi \Lambda^{s} w .
$$

The following lemma allows us to finish the proof of the proposition.

Lemma 11. The operator $T_{\phi} w$ defined by

$$
\begin{aligned}
T_{\phi}: \quad L_{u l o c}^{2} & \longrightarrow L_{u l o c}^{2} \\
w & \longmapsto\left[\Lambda^{s}, \phi\right] w
\end{aligned}
$$

is continuous.

Proof. We write

$$
\begin{aligned}
\left|\left[\Lambda^{s}, \phi\right] w\right| & \leq C_{s} \int \frac{|\phi(x)-\phi(y)|}{|x-y|^{2+s}}|w(y)| d y \\
& \leq C_{s} \int \frac{\min \left(\|\phi\|_{\infty},\|\nabla \phi\|_{L^{\infty}}|x-y|\right)}{|x-y|^{2+s}}|w(y)| d y \\
& \leq C_{s} \int \frac{\min (1,|x-y|)}{|x-y|^{2+s}}|w(y)| d y \\
& =(A *|w|)(x),
\end{aligned}
$$

where $A(x)=C_{s} \frac{\min (1,|x|)}{|x|^{2+s}}$. Then, we see that if $|x|<1$ then $A(x)=\frac{C_{s}}{|x|^{1+s}} \in L^{1}$ and since $|w| \in L_{u l o c}^{2}$ by convolution (see Lemma 2) we get that $A *|w| \in L_{\text {uloc }}^{2}$

If $|x|>1$ then $A(x)=\frac{C_{s}}{|x|^{2+s}} \in L^{1}$ and the same conclusion holds and this finishes the proof of the lemma.

Thus, $\psi\left[\Lambda^{s}, \phi\right] w \in L^{2}$. For the term $(1-\psi)\left[\Lambda^{s}, \phi\right] w$ we see that:

$$
\begin{aligned}
(1-\psi)\left[\Lambda^{s}, \phi\right] w & =(1-\psi) \Lambda(\phi w)-(1-\psi) \phi \Lambda w \\
& =(1-\psi)\left(C_{s} \frac{\mathbb{1}_{x \geq 1}}{|x|^{2+s}} * w \phi\right) .
\end{aligned}
$$

The kernel $\frac{C_{s} \mathbb{1}_{x \geq 1}}{|x|^{2+s}}$ is in $L^{1}$, and since $w \phi \in L^{2}$, by convolution, we get that $(1-\psi)\left[\Lambda^{s}, \phi\right] w \in L^{2}$ and this ends the proof of the proposition.

\section{$3 \quad$ The energy inequality}

\subsection{Well known existence results in the critical case.}

In this section, we recall some existence and uniqueness results that we will use in the proof of our main theorem. Let us recall that the existence of global weak solutions of $(S Q G)$ with initial data in $L^{2}\left(\mathbb{R}^{2}\right)$ has been obtained by Resnick in [18]. More precisely, he proved the following theorem: 
Theorem 8 (Resnick). Let $\theta_{0} \in L^{2}\left(\mathbb{R}^{2}\right)$. Then, for any $T>0$, there exists at least one weak solution to the critical ( $S Q G$ ) equation in the following sense:

$$
\partial_{t} \int \theta \phi d x-\int \theta(u . \nabla \phi) d x+\int\left(\Lambda^{1 / 2} \phi\right)\left(\Lambda^{1 / 2} \theta\right) d x=0, \quad \forall \phi \in \mathcal{D}
$$

Moreover,

$$
\theta \in L^{\infty}\left([0, T], L^{2}\left(\mathbb{R}^{2}\right)\right) \cap L^{2}\left([0, T], \dot{H}^{1 / 2}\left(\mathbb{R}^{2}\right)\right) .
$$

From the result of Caffarelli and Vasseur [4], those weak solutions are known to be smooth. Unfortunately, the smoothness is not sufficient in our proof since in our future computations we need a little bit of integrability (at least $H^{1}$ ) therefore we need a result of global and regular enough solutions without condition of smallness on the initial data. As we recalled in the introduction, Abidi and Hmidi in [1] proved a theorem of global solution for large data in the critical Besov space $\theta_{0} \in \dot{B}_{\infty, 1}^{0}$. Let us recall their main theorem; to do so, we need to recall the definition of the homogenous Besov spaces based on the classical Littlewood-Paley decomposition.

Let $\phi$ be a smooth function supported in the $\operatorname{ring} \mathcal{C}:=\left\{\xi \in \mathbb{R}^{2}, \frac{3}{4} \leq|\xi| \leq \frac{8}{3}\right\}$ and such that

$$
\sum_{q \in \mathbb{Z}} \phi\left(2^{-q} \xi\right)=1 \quad \text { for } \quad \xi \neq 0
$$

Now, for $u \in \mathcal{S}^{\prime}$ we set

$$
\forall q \in \mathbb{Z}, \quad \Delta_{q} u=\phi\left(2^{-q} \mathrm{D}\right) u \quad \text { and } \quad S_{q} u=\sum_{j \leq q-1} \Delta_{j} u .
$$

Recall that we have the following formal Littlewood-Paley decompostion of $u$

$$
u=\sum_{q \in \mathbb{Z}} \Delta_{q} u, \quad \forall u \in \mathcal{S}^{\prime}\left(\mathbb{R}^{2}\right) / \mathcal{P}\left[\mathbb{R}^{2}\right]
$$

where $\mathcal{P}\left[\mathbb{R}^{2}\right]$ is the set of polynomials.

Now we are able to give the definition of the homogenous Besov spaces. Let $(p, m) \in[1,+\infty]^{2}$, $s \in \mathbb{R}$ and $u \in \mathcal{S}^{\prime}, u$ is said to be in $\dot{\mathcal{B}}_{p, m}^{s}$ if

$$
\|u\|_{\dot{\mathcal{B}}_{p, m}^{s}}:=\left(2^{q s}\left\|\Delta_{q} u\right\|_{L^{p}}\right)_{\ell^{m}}<\infty
$$

In [1], Abidi and Hmidi proved the following regularity theorem:

Theorem 9 (Abidi and Hmidi). Let $\theta_{0} \in \dot{B}_{\infty, 1}^{0}$, then there exists a unique global solution $\theta$ to the dissipative critical $S Q G$ equation such that

$$
\theta \in \mathcal{C}\left(\mathbb{R}_{+} ; \dot{B}_{\infty, 1}^{0}\right) \cap L_{\text {loc }}^{1}\left(\mathbb{R}_{+} ; \dot{B}_{\infty, 1}^{1}\right)
$$

Remark 12. The space $\dot{B}_{\infty, 1}^{0}$ is defined as the completion in the Schwartz class for the norm $L^{\infty}$ with respect to the norm $\|u\|_{\dot{\mathcal{B}}_{\infty, 1}^{0}}=\sum_{q \in \mathbb{Z}}\left\|\Delta_{q} u\right\|_{L^{\infty}}$.

We recall the well-known result due to A. Córdoba and D. Córdoba [10].

Lemma 13. Let $0 \leq \alpha \leq 2$, the following pointwise inequality holds for all convex functions $g$,

$$
\Lambda^{\alpha}(g(\theta)) \leq g^{\prime}(\theta) \Lambda^{\alpha}(\theta)
$$

Proof. See e.g. [10].

We shall use the maximum principle for the (SQG) equation due to Resnick [18] and also A.Córdoba and D.Córdoba [10], that is: 
Lemma 14 (maximum principle). Let $\theta$ be a smooth function satisfying the $(S Q G)_{\alpha}$ equation $\partial_{t} \theta+u . \nabla \theta+k \Lambda^{\alpha} \theta=0$, where $0 \leq \alpha \leq 2$ and $k \geq 0$, then we have for all $p \in[2,+\infty)$ and for all $t \geq 0$

$$
\|\theta\|_{L^{p}}^{p}+2 k \int_{0}^{t} \int\left|\Lambda^{\alpha / 2}\left(|\theta|^{p / 2}\right)\right|^{2} d x d s \leq\left\|\theta_{0}\right\|_{L^{p}}^{p}
$$

and

$$
\|\theta(t)\|_{L^{\infty}} \leq\left\|\theta_{0}\right\|_{L^{\infty}}
$$

Proof. See e.g. [10].

\subsection{The critical SQG equation with $\theta=\Lambda^{s} w$}

Let us recall that we want to give some oscillations to $\theta$, so we put $\theta=\Lambda^{s} w=(-\Delta)^{s / 2} w$ and we suppose that $w \in H_{u l o c}^{s}$ and that $\theta \in L^{\infty}$. The (SQG) equation becomes:

$$
\Lambda^{s}\left(\partial_{t} w\right)=\nabla \cdot\left(\Lambda^{s} w\left(\mathcal{R}^{\perp} \Lambda^{s} w\right)\right)-\Lambda^{s+1} w
$$

hence, we get

$$
\partial_{t} w=\left(\Lambda^{-s} \nabla\right) \cdot\left(\Lambda^{s} w\left(\mathcal{R}^{\perp} \Lambda^{s} w\right)\right)-\Lambda w
$$

The equation that we will study through this paper is the next one:

$$
(S \tilde{Q} G):\left\{\begin{array}{l}
\partial_{t} w=\left(\Lambda^{-s} \nabla\right) \cdot\left(\Lambda^{s} w\left(\mathcal{R}^{\perp} \Lambda^{s} w\right)\right)-\Lambda w \\
\nabla \cdot \mathcal{R}^{\perp} \Lambda^{s} w=0 .
\end{array}\right.
$$

Note that the initial value problem is now endowed with the conditions:

$$
\theta_{0}(x)=\Lambda^{s} w_{0} \in \Lambda^{s}\left(H_{u l o c}^{s}\right) \cap L^{\infty} .
$$

Before we go any further, we have to check that the $(S \widetilde{Q} G)$ equation makes sense when $w \in$ $L^{\infty}\left([0, T], \dot{H}_{u l o c}^{s}\left(\mathbb{R}^{2}\right)\right)$ and $\Lambda^{s} w \in L^{\infty}\left([0, T], L^{\infty}\left(\mathbb{R}^{2}\right)\right)$ where $T>0$. The equation $(S \tilde{Q} G)$ is:

$$
\partial_{t} w=\Lambda^{-s} \nabla \cdot \Lambda^{s} w\left(\mathcal{R}^{\perp} \Lambda^{s} w\right)-\Lambda w
$$

If $w \in L^{\infty}\left([0, T], H_{u l o c}^{s}\left(\mathbb{R}^{2}\right)\right)$ then the computation $\Lambda w$ has obviously a sense in $\mathcal{D}^{\prime}$ and $\Lambda^{s} w \in$ $L^{\infty}\left([0, T], L_{u l o c}^{2}\left(\mathbb{R}^{2}\right)\right)$. We now deal with the non linear term which is:

$$
\Lambda^{-s} \nabla \cdot \Lambda^{s} w\left(\mathcal{R}^{\perp} \Lambda^{s} w\right)
$$

We begin with the study of the singular integral operator $\mathcal{R}^{\perp} \Lambda^{s}$. Recalling that $\mathcal{R}^{\perp} \theta=\nabla^{\perp} \Lambda^{-1} \theta$, we infer $\mathcal{R}^{\perp} \theta=\nabla^{\perp} \Lambda^{s-1} w=\left(-\partial_{2} \Lambda^{s-1} w, \partial_{1} \Lambda^{s-1} w\right)$, since the convolution kernel of the operator $\Lambda^{s-1} \theta$ is defined by $K(x)=\frac{C_{s}}{|x|^{1+s}}$; therefore, we see that the convolution kernel $\tilde{K}$ of $u_{R}$ is given by:

$$
\tilde{K}(x)=\partial_{j} \frac{C_{s}}{|x|^{1+s}}=\frac{-C_{s}(1+s)}{|x|^{2+s}} \frac{x_{j}}{|x|} .
$$

Now, let $\alpha$ be a smooth cut-off function which is equal to one for $|x| \leq 1$ and zero for $|x| \geq 2$. Then we split,

$$
u_{R}=\alpha \tilde{K} * w+(1-\alpha) \tilde{K} * w .
$$

Since $\tilde{K} \alpha \in \mathcal{E}_{\tilde{K}}^{\prime}$ and $w \in L_{u l o c}^{2} \subset \mathcal{D}^{\prime}$ the convolution makes sense. For the second part, we notice that $(1-\alpha) \tilde{K} * \theta \in L^{1} * L_{\text {uloc }}^{2} \subset \mathcal{D}^{\prime}$.

The idea of the proof of our main result is the following. We introduce a truncated initial data:

$$
\theta_{0, R}=\Lambda^{s}\left(w_{0} \chi_{R}\right)
$$


where $\chi_{R}$ is a positive smooth function constructed as follows. Let $\chi \in \mathcal{D}\left(\mathbb{R}^{2}\right)$ be a positive smooth function s.t $\chi(x)=1$ if $|x| \leq 1$, and 0 if $|x| \geq 2$. For $R>0$, we introduce the function $\chi_{R}(x) \equiv \chi(x / R)$. By construction, the function $\chi_{R}$ is a positive smooth function s.t for all $R>0$,

$$
\left\{\begin{array}{l}
\chi_{R}(x)=1 \text { if }|x| \leq R \\
\chi_{R}(x)=0 \text { if }|x| \geq 2 R
\end{array}\right.
$$

We also need to introduce the following standard mollifier $\rho \in \mathcal{D}\left(\mathbb{R}^{2}\right)$, s.t supp $\rho \subset[-1,1]^{2}$ and $\int_{\mathbb{R}^{2}} \rho=1$, then we define $\rho_{\epsilon}(x)=\frac{1}{\epsilon^{2}} \rho\left(\frac{x}{\epsilon}\right)$.

We will study the following truncated equation associated with a truncated and regularized initial data.

$$
\left\{\begin{array}{l}
\partial_{t} w_{R}=\left(\Lambda^{-s} \nabla\right) \cdot\left(\Lambda^{s} w_{R} \mathcal{R}^{\perp} \Lambda^{s} w_{R}\right)-\Lambda w_{R} \\
\nabla \cdot \mathcal{R}^{\perp} \Lambda^{s} w_{R}=0 \\
\theta_{0, R, \epsilon}=\Lambda^{s}\left(w_{0} \chi_{R}\right) * \rho_{\epsilon}
\end{array}:(S Q G)_{R, \epsilon}\right.
$$

Since the initial data $\theta_{0} \in \Lambda^{s}\left(H_{\text {uloc }}^{s}\left(\mathbb{R}^{2}\right)\right) \cap L^{\infty}\left(\mathbb{R}^{2}\right)$ then the truncated initial data $\theta_{0, R} \in$ $L^{2}\left(\mathbb{R}^{2}\right) \cap L^{\infty}\left(\mathbb{R}^{2}\right)$ and

$$
\theta_{0, R, \epsilon} \in \bigcap_{k \geq 0} H^{k} \subset H^{2} \subset \dot{B}_{\infty, 1}^{0}
$$

Therefore, we can use the result of Abidi and Hmidi [1] and claim that for the truncated and regularized initial data, there exists at least one global weak solution $w_{R}$ to the $(S Q G)_{R, \epsilon}$ equation. Moreover, the solution belongs to the following space:

$$
\theta_{R} \in \mathcal{C}\left(\mathbb{R}_{+} ; \dot{B}_{\infty, 1}^{0}\right) \cap L_{\mathrm{loc}}^{1}\left(\mathbb{R}_{+} ; \dot{B}_{\infty, 1}^{1}\right)
$$

Then, we will obtain an energy inequality involving the truncated solution and using some estimates and compactness arguments, we will show that we can pass to the weak limit of the $(S Q G)_{R, \epsilon}$ equation. The solution of the $(S Q G)$ equation will be the weak limit of $w_{R, \epsilon}$.

The following lemma will allow us to apply the maximum principle to $w_{0}$.

Lemma 14. If $w \in H_{u l o c}^{s}\left(\mathbb{R}^{2}\right)$ and $\theta=\Lambda^{s} w \in L^{\infty}\left(\mathbb{R}^{2}\right)$ then $w \in L^{\infty}\left(\mathbb{R}^{2}\right)$

Proof. We decompose the operator $\Lambda^{s}$ in low frequencies and high frequencies. We denote $S_{0}$ the low frequencies and we write:

$$
w=\Lambda^{-s}\left(I d-S_{0}\right) \Lambda^{s} w+S_{0} w .
$$

Since the operator $\Lambda^{-s}\left(I d-S_{0}\right)$ is continuous from $L^{\infty}$ to $L^{\infty}$ thus $\Lambda^{-s}\left(I d-S_{0} w\right) \Lambda^{s} w \in L^{\infty}$. Moreover, since $H_{u l o c}^{s}\left(\mathbb{R}^{2}\right)$ is a shift invariant space, one can easily see that $S_{0} w \in L^{\infty}$.

\subsection{Bounds for the truncated and regularized initial data}

Now, we have to check that the truncated and regularized initial data lies in $L^{\infty}$ uniformly with respect to the parameters $R$ and $\epsilon$.

Lemma 15. If $\theta_{0} \in L^{\infty}$ and $w_{0} \in H_{u l o c}^{s}\left(\mathbb{R}^{2}\right)$ then $\sup _{R>1, \epsilon>0}\left\|\theta_{0, R, \epsilon}\right\|_{L^{\infty}\left(\mathbb{R}^{2}\right)}<\infty$

Proof. Using a commutator, one can write

$$
\theta_{0, R, \epsilon}=\Lambda^{s}\left(w_{0} \chi_{R}\right) * \rho_{\epsilon}=\left(\chi_{R} \Lambda^{s} w_{0}+\int \frac{w_{0}(y)\left(\chi_{R}(x)-\chi_{R}(y)\right)}{|x-y|^{2+s}} d y\right) * \rho_{\epsilon} .
$$


Since $\Lambda^{s} w_{0} \in L^{\infty}$ then $\chi_{R} \Lambda^{s} w_{0} \in L^{\infty}$. Now, we have to check that the integral in the bracket lies in $L^{\infty}$ uniformly in $R$. For all $R>1$, we split and use the Young inequality $\left(L^{\infty} * L^{1} \subset L^{\infty}\right)$ to get:

$$
\begin{aligned}
& \int \frac{w_{0}(y)\left(\chi_{R}(x)-\chi_{R}(y)\right)}{|x-y|^{2+s}} d y \\
& =\int_{|x-y|<R} \frac{w_{0}(y)\left(\chi_{R}(x)-\chi_{R}(y)\right)}{|x-y|^{2+s}} d y+\int_{|x-y|>R} \frac{w_{0}(y)\left(\chi_{R}(x)-\chi_{R}(y)\right)}{|x-y|^{2+s}} d y \\
& \leq \int_{|x-y|<R} \frac{w_{0}(y)\left\|\nabla \chi_{R}\right\|_{L^{\infty}}}{|x-y|^{1+s}} d y+2 \int_{|x-y|>R} \frac{w_{0}(y)\left\|\chi_{R}\right\|_{L^{\infty}}}{|x-y|^{2+s}} d y \\
& \leq\left\|\nabla \chi_{R}\right\|_{L^{\infty}} \int_{|y|<R} \frac{w_{0}(x-y)}{|y|^{1+s}} d y+2\left\|\chi_{R}\right\|_{L^{\infty}} \int_{|y|>R} \frac{w_{0}(x-y)}{|y|^{2+s}} d y .
\end{aligned}
$$

We thus get,

$$
\left\|\int \frac{w_{0}(y)\left(\chi_{R}(x)-\chi_{R}(y)\right)}{|x-y|^{2+s}} d y\right\|_{L^{\infty}} \leq R^{-s}\|\nabla \chi\|_{L^{\infty}}\left\|w_{0}\right\|_{L^{\infty}}+2 R^{-s}\left\|\chi_{R}\right\|_{L^{\infty}}\left\|w_{0}\right\|_{L^{\infty}}
$$

Then using the Young inequality and taking the supremum over $R>1$ and $\epsilon>0$ yields

$$
\sup _{R>1, \epsilon>0}\left\|\theta_{0, R, \epsilon>0}\right\|_{L^{\infty}}=\sup _{R>1, \epsilon>0}\left\|\Lambda^{s}\left(w_{0} \chi_{R}\right) * \rho_{\epsilon}\right\|_{L^{\infty}} \leq \sup _{R>1, \epsilon>0}\left\|\theta_{0, R}\right\|_{L^{\infty}}\left\|\rho_{\epsilon}\right\|_{L^{1}} \lesssim \sup _{R>1}\left\|\theta_{0, R}\right\|_{L^{\infty}}
$$

We also need the following uniform control on the truncated and regularized initial data.

Lemma 16. If $\theta_{0} \in L^{\infty}$ and $w_{0} \in H_{u l o c}^{s}\left(\mathbb{R}^{2}\right)$ then $\sup _{R>1, \epsilon>0}\left\|w_{0, R, \epsilon}\right\|_{H_{u l o c}^{s}\left(\mathbb{R}^{2}\right)}<\infty$

Proof. It is equivalent to show that, for all $R>1$ and $\epsilon>0, \Lambda^{s} w_{0, R, \epsilon} \in L_{u l o c}^{2}$. As in the previous proof, we write

$$
\Lambda^{s}\left(w_{0} \chi_{R}\right) * \rho_{\epsilon}=\left(\chi_{R} \Lambda^{s} w_{0}+C_{s} \int \frac{w_{0}(y)\left(\chi_{R}(x)-\chi_{R}(y)\right)}{|x-y|^{2+s}} d y\right) * \rho_{\epsilon}
$$

Since $w_{0} \in H_{u l o c}^{s}\left(\mathbb{R}^{2}\right)$ then $\Lambda^{s} w_{0} \in L_{u l o c}^{2}$ and for all $R>1$, we see that $\chi_{R} \Lambda^{s} w_{0, R} \in L_{u l o c}^{2}$.

For the second term, we write

$$
\begin{aligned}
& \int \frac{w_{0}(y)\left(\chi_{R}(x)-\chi_{R}(y)\right)}{|x-y|^{2+s}} d y \\
& =\int_{|x-y|<R} \frac{w_{0}(y)\left(\chi_{R}(x)-\chi_{R}(y)\right)}{|x-y|^{2+s}} d y+\int_{|x-y|>R} \frac{w_{0}(y)\left(\chi_{R}(x)-\chi_{R}(y)\right)}{|x-y|^{2+s}} d y \\
& \leq \int_{|x-y|<R} \frac{\left\|\nabla \chi_{R}\right\|_{L^{\infty}} w_{0}(y)}{|x-y|^{1+s}} d y+2 \int_{|x-y|>R} \frac{\left\|\chi_{R}\right\|_{L^{\infty}} w_{0}(y)}{|x-y|^{2+s}} d y \\
& \leq R^{-s}\|\nabla \chi\|_{L^{\infty}} \int_{|y|<R} \frac{w_{0}(x-y)}{|y|^{1+s}} d y+2\left\|\chi_{R}\right\|_{L^{\infty}} \int_{|y|>R} \frac{w_{0}(x-y)}{|y|^{2+s}} d y .
\end{aligned}
$$

Thus we obtain

$$
\left\|C_{s} \int \frac{w_{0}(y)\left(\chi_{R}(x)-\chi_{R}(y)\right)}{|x-y|^{2+s}} d y\right\|_{L_{u l o c}^{2}} \lesssim\left(R^{-s}\|\nabla \chi\|_{L^{\infty}}+2\left\|\chi_{R}\right\|_{L^{\infty}}\right)\left\|w_{0}\right\|_{L_{u l o c}^{2}\left(\mathbb{R}^{2}\right)}
$$

where we have use the fact that, since $w_{0} \in H_{u l o c}^{s}\left(\mathbb{R}^{2}\right)$ then $w_{0} \in L_{u l o c}^{2}\left(\mathbb{R}^{2}\right)$ and $\mathbb{1}_{|y|<R} \frac{1}{|y|^{1+s}} \in L^{1}$ then $\int \frac{w_{0}(y)}{|x-y|^{1+s}} d y \in L_{u l o c}^{2}$. 
For the second integral, we use that $\mathbb{1}_{|y|>R} \frac{1}{|y|^{2+s}} \in L^{1}$ and since $w_{0} \in L_{u l o c}^{2}$ by convolution we conclude that $\int_{|y|>R} \frac{w_{0}(x-y)}{|y|^{1+s}} d y \in L_{u l o c}^{2}$.

Now, we write

$$
\begin{aligned}
\sup _{R>1, \epsilon>0}\left\|w_{0, R, \epsilon}\right\|_{H_{u l o c}^{s}\left(\mathbb{R}^{2}\right)}=\sup _{R>1, \epsilon>0}\left\|w_{0, R} * \rho_{\epsilon}\right\|_{H_{u l o c}^{s}\left(\mathbb{R}^{2}\right)} & \leq \sup _{R>1, \epsilon>0}\left\|w_{0, R}\right\|_{H_{u l o c}^{s}\left(\mathbb{R}^{2}\right)}\left\|\rho_{\epsilon}\right\|_{L^{1}} \\
& \lesssim \sup _{R>1}\left\|w_{0, R}\right\|_{H_{u l o c}^{s}\left(\mathbb{R}^{2}\right)}
\end{aligned}
$$

\subsection{Energy inequality and uniform bounds}

In this section, we prove the following theorem:

Theorem 17. Suppose that $1 / 2<s<1$, and that $w_{R, \epsilon}$ is a weak solution of $(S Q G)_{R, \epsilon}$, then we have the following energy inequality

$$
\partial_{t} A_{\phi}\left(w_{R, \epsilon}\right) \leq C\left\|w_{R, \epsilon}\right\|_{H_{u l o c}^{s}}^{2}\left(\mathbb{R}^{2}\right)
$$

where $C$ is a constant depending only on $\left\|\theta_{0}\right\|_{L^{\infty}\left(\mathbb{R}^{2}\right)}$ and $\left\|w_{0}\right\|_{H_{u l o c}^{s}\left(\mathbb{R}^{2}\right)}$.

Proof. Let $w_{R, \epsilon}$ be a weak solution to $(S Q G)_{R, \epsilon}$ equation. Recall that,

$$
\left\|w_{R, \epsilon}\right\|_{H_{u l o c}^{s}}^{2}\left(\mathbb{R}^{2}\right)=\sup _{\phi \in B} \int\left(\frac{\left|w_{R, \epsilon}\right|^{2}}{2} \phi+\frac{\left|\Lambda^{s} w_{R, \epsilon}\right|^{2}}{2} \phi\right) d x=\sup _{\phi \in B} A_{\phi}\left(w_{R, \epsilon}\right) .
$$

We have

$$
\begin{aligned}
\partial_{t} A_{\phi}\left(w_{R, \epsilon}\right) & =\int \partial_{t}\left(\frac{\left|w_{R, \epsilon}\right|^{2}}{2} \phi+\frac{\left|\Lambda^{s} w_{R, \epsilon}\right|^{2}}{2} \phi\right) d x \\
& =\int w_{R, \epsilon} \phi \partial_{t} w_{R, \epsilon}+\phi \Lambda^{s} w_{R, \epsilon} \Lambda^{s}\left(\partial_{t} w_{R, \epsilon}\right) d x .
\end{aligned}
$$

We have to justify that the first equality makes sense in $\mathcal{D}^{\prime}\left((0, T] \times \mathbb{R}^{2}\right)$. Let us remark that, since $w$ is more regular than $\theta$, it is enough to show that $\int \phi \theta_{R, \epsilon} \partial_{t} \theta_{R, \epsilon} d x$ makes sense. We begin with the regularity of $\partial_{t} \theta_{R, \epsilon}$, using the equation we have

$$
\partial_{t} \theta_{R, \epsilon}=-\nabla \cdot\left(\theta_{R, \epsilon} u_{R, \epsilon}\right)-\Lambda \theta_{R, \epsilon}
$$

Since $\theta_{R, \epsilon} \in L^{2} \dot{H}^{1 / 2}$ then $\Lambda \theta_{R, \epsilon} \in L^{2} \dot{H}^{-1 / 2}$, moreover, by the continuity of the Riesz transforms on $L^{2} \dot{H}^{1 / 2}$ we infer $u_{R, \epsilon} \in L^{2} \dot{H}^{1 / 2}$. We would like to have $u_{R} \theta_{R} \in L^{2} \dot{H}^{1 / 2}$. Since we have

$$
\left\|u_{R, \epsilon} \theta_{R, \epsilon}\right\|_{\dot{H}^{1 / 2}} \lesssim\left\|u_{R, \epsilon}\right\|_{L^{\infty}}\left\|\theta_{R, \epsilon}\right\|_{\dot{H}^{1 / 2}}+\left\|u_{R, \epsilon}\right\|_{\dot{H}^{1 / 2}}\left\|\theta_{R, \epsilon}\right\|_{L^{\infty}},
$$

it suffices to show that $u_{R, \epsilon} \in L^{\infty} L^{\infty}$. Indeed, using the Abidi and Hmidi result, we infer that the solutions we obtain are in the space $L^{\infty} \dot{B}_{\infty, 1}^{0}$. Since the Riesz transforms map continuously the space $L^{\infty} \dot{B}_{\infty, 1}^{0}$ into itself, and thanks to the embedding $L^{\infty} \dot{B}_{\infty, 1}^{0} \hookrightarrow L^{\infty} L^{\infty}$, we get that $u_{R, \epsilon} \in L^{\infty} L^{\infty}$. Therefore, $u_{R, \epsilon} \Lambda^{s} w_{R, \epsilon} \in L^{2} \dot{H}^{1 / 2}$, which we had not before regularized since the Riesz transforms are not continuous on the space $L^{\infty} L^{\infty}$. Then, we have $\nabla .\left(\theta_{R, \epsilon} u_{R, \epsilon}\right) \in L^{2} \dot{H}^{-1 / 2}$ and we conclude that $\partial_{t} \theta \in L^{2} \dot{H}^{-1 / 2}$. Since $\phi \theta_{R, \epsilon} \in L^{2} \dot{H}^{1 / 2}$, then the term $\int \phi \theta_{R, \epsilon} \partial_{t} \theta_{R, \epsilon} d x$ makes sense and we have:

$$
\partial_{t} A_{\phi}\left(w_{R, \epsilon}\right)=\int w_{R, \epsilon} \phi \partial_{t} w_{R, \epsilon} d x+\int \phi \Lambda^{s} w_{R, \epsilon} \Lambda^{s}\left(\partial_{t} w_{R, \epsilon}\right) d x
$$


Replacing $\partial_{t} w_{R, \epsilon}$ by $-\left(\Lambda^{-s} \nabla\right) \cdot\left(\Lambda^{s} w_{R, \epsilon}\left(\mathcal{R}^{\perp} \Lambda^{s} w_{R, \epsilon}\right)\right)-\Lambda w_{R, \epsilon}$ in (3) leads us to the following equality:

$$
\begin{aligned}
\partial_{t} A_{\phi}\left(w_{R, \epsilon}\right)=-\int w_{R, \epsilon} \phi \Lambda^{-s} \nabla & \left(u \Lambda^{s} w_{R, \epsilon}\right) d x-\int w_{R, \epsilon} \phi \Lambda w_{R, \epsilon} d x \\
& -\int \phi \Lambda^{s} w_{R, \epsilon} \nabla \cdot\left(u_{R, \epsilon} \Lambda^{s} w_{R, \epsilon}\right) d x-\int \phi \Lambda^{s} w_{R, \epsilon} \Lambda^{s+1} w_{R, \epsilon} d x .
\end{aligned}
$$

In the estimation of the two first terms, we use the fact that the Riesz transforms $u_{R, \epsilon}$ are controlled in the space $L^{\infty} L_{u l o c}^{2}$, more precisely we have the following lemma.

Lemma 18. $u_{R, \epsilon}$ is bounded in $L_{t}^{2} L_{u l o c}^{2}$ and we have the following estimate:

$$
\left\|u_{R, \epsilon}\right\|_{\left(L_{t}^{2} L^{2}\right)_{u l o c}} \lesssim\left\|w_{R, \epsilon}\right\|_{L_{t}^{2} H_{u l o c}^{s}} .
$$

Proof. It is equivalent to show that for all $\psi \in B_{\psi}, \psi u_{R, \epsilon}$ is uniformly controlled in $L_{t}^{2} L^{2}$. We split $\psi u_{R, \epsilon}$ as follows:

$$
\begin{aligned}
\psi u_{R, \epsilon}=\psi \mathcal{R}^{\perp} \Lambda^{s} w_{R, \epsilon} & =\psi \mathcal{R}^{\perp} \Lambda^{s}\left(\phi w_{R, \epsilon}\right)+\psi \mathcal{R}^{\perp} \Lambda^{s}(1-\phi) w_{R, \epsilon} \\
& =(1)+(2) .
\end{aligned}
$$

Since $w_{R, \epsilon} \in\left(L^{2} H^{s}\right)_{u l o c}$ then $\phi w_{R, \epsilon} \in L_{t}^{2} H^{s}$ and $\Lambda^{s}\left(\phi w_{R, \epsilon}\right) \in L_{t}^{2} L^{2}$, by the continuity of the Riesz operator on $L_{t}^{2} L^{2}$ we deduce that $\mathcal{R}^{\perp} \Lambda^{s}\left(\phi w_{R, \epsilon}\right)$ is controlled in $L_{t}^{2} L^{2}$ and (1) as well. Moreover we have the following inequalities:

$$
\begin{aligned}
\left\|\mathcal{R}^{\perp} \Lambda^{s}\left(\phi w_{R, \epsilon}\right)\right\|_{L_{t}^{2} L^{2}} \leq\left\|\Lambda^{s}\left(\phi w_{R, \epsilon}\right)\right\|_{L_{t}^{2} L^{2}} & \leq \sup _{\phi \in B}\left\|\Lambda^{s}\left(\phi w_{R, \epsilon}\right)\right\|_{L_{t}^{2} L^{2}} \\
& \leq\left\|w_{R, \epsilon}\right\|_{L_{t}^{2} \dot{H}_{u l o c}^{s}} .
\end{aligned}
$$

Let us denote by $\tilde{K}$ the kernel of the operator $\mathcal{R}^{\perp} \Lambda^{s}$. We have previously seen that this kernel behaves like $\frac{C_{s}}{|x|^{2+s}}$ which is in $L^{1}$ far from the origin, since $w_{R, \epsilon} \in L_{t}^{2} L_{u l o c}^{2}$ we get

$$
\begin{aligned}
\left\|\mathcal{R}^{\perp} \Lambda^{s}(1-\phi) w_{R, \epsilon}\right\|_{L_{t}^{2} L_{u l o c}^{2}} & \leq\left\|\mathbb{1}_{x \in(\operatorname{supp} \phi)^{c}} \tilde{K} * \phi w_{R, \epsilon}\right\|_{L_{t}^{2} L_{u l o c}^{2}} \\
& \leq\left\|\mathbb{1}_{x \in(\text { supp } \phi)^{c}} \tilde{K}\right\|_{L^{1}}\left\|\phi w_{R, \epsilon}\right\|_{L^{2} L_{\text {uloc }}^{2}} \\
& \lesssim\left\|w_{R, \epsilon}\right\|_{L^{2} H_{u l o c}^{s}} .
\end{aligned}
$$

Let us estimate each of those four terms; to avoid notational burden, we will omit to write the depedence on $\epsilon$. For the first one, we would like to write:

$$
-\int \Lambda^{-s} \nabla \cdot\left(w_{R} \phi\right) u_{R} \Lambda^{s} w_{R} d x d x \leq\left\|\Lambda^{-s} \nabla \cdot\left(w_{R} \phi\right)\right\|_{L_{u l o c}^{2}}\left\|u_{R}\right\|_{L_{u l o c}^{2}}\left\|\theta_{R}\right\|_{L^{\infty}} .
$$

This is done if and only if we have a negative Sobolev regularity on the term $\Lambda^{-s} \nabla \cdot\left(w_{R} \phi\right)$ since we cannot control $u_{R}$ in a space of positive Sobolev regularity (uniformly with respect to the parameters $R$ and $\epsilon$ ). Indeed, controlling $u_{R}$ in a space of positive Sobolev regularity means that we can get a control of the low frequencies of $u_{R}$ which is hopeless. This is due to the fact that since $\theta_{R} \in L^{\infty} L^{\infty}$ then $u_{R} \in L^{\infty} B M O$ and, in particular, we cannot get a control of $\phi u_{R}$ because $\phi u_{R} \notin B M O$ ( $B M O$ is a space which is defined only modulo constants). Since $\phi w_{R} \in L^{2} \dot{H}^{s+1 / 2}$ then $\Lambda^{-s} \nabla .\left(w_{R} \phi\right) \in L^{2} \dot{H}^{2 s-1 / 2}$ we thus need $2 s-1 / 2>0$ that is $1 / 4 \leq s \leq 1$. Then, we can write

$$
\begin{aligned}
-\int \Lambda^{-s} \nabla \cdot\left(w_{R} \phi\right) u_{R} \Lambda^{s} w_{R} d x d x & \leq\left\|\Lambda^{-s} \nabla \cdot\left(w_{R} \phi\right)\right\|_{L_{u l o c}^{2}}\left\|u_{R}\right\|_{L_{u l o c}^{2}}\left\|\theta_{R}\right\|_{L^{\infty}} \\
& \lesssim\left\|\phi w_{R}\right\|_{\dot{H}_{u l o c}^{1-s}}^{1-s}\left\|w_{R}\right\|_{H_{u l o c}^{s}}\left\|\theta_{0, R}\right\|_{L^{\infty}} \\
& \lesssim \sup _{\phi \in B_{\phi_{0}}}\left\|\phi w_{R}\right\|_{\dot{H}_{u l o c}^{s}}\left\|w_{R}\right\|_{H_{u l o c}^{s}}\left\|\theta_{0, R}\right\|_{L^{\infty}} \\
& \lesssim\left\|w_{R}\right\|_{\dot{H}_{u l o c}^{s}}^{2}
\end{aligned}
$$


where we used the embedding $H^{s} \subset H^{1-s}$ for $1 / 2<s<1$.

For the second term, we use the following decay property:

Lemma 19. Let $\phi \in \mathcal{D}\left(\mathbb{R}^{2}\right)$ then, for all $x \in \mathbb{R}^{2}$ we have $|\Lambda \phi(x)| \leq \frac{C}{|x|^{3}}$ where $C$ is a fixed positive constant.

Proof. Let $B_{R}$ denote the ball on $\mathbb{R}^{2}$ of radius $R>0$ centered at the origin. Suppose that $\phi \in \mathcal{D}\left(\mathbb{R}^{2}\right)$ is such that

$$
\left\{\begin{array}{l}
\phi(x)=1 \text { on } B_{R} \\
\phi(x)=0 \text { on } B_{R+1}^{c}
\end{array}\right.
$$

If $x \in B_{R+2}^{c}$ then

$$
|\Lambda \phi(x)| \leq \int_{y \in B_{R}} \frac{|\phi(y)|}{|x-y|^{3}} d y \leq \frac{C}{|x|^{3}}
$$

If $x \in B_{R}$, and $y \in B_{R+2}^{c}$ it is the same case as before by symmetry. Now, for $x \in B_{R}$ and $y \in B_{R}$, we use the following representation of the fractional Laplacian; since $\phi \in \mathcal{S}$ one has

$$
\Lambda \phi=-\frac{1}{2} \int \frac{\phi(x+y)+\phi(x-y)-2 \phi(x)}{|y|^{3}} d y .
$$

Using a second order Taylor expansion makes us get rid of the singularity at the origin. More precisely, one has

$$
\frac{\phi(x+y)+\phi(x-y)-2 \phi(x)}{|y|^{3}} \leq \frac{\left\|\nabla^{2} \phi\right\|_{L^{\infty}}}{|y|}
$$

Since the right-hand side of the previous inequality is in $L^{1}\left(B_{R}\right)$, we get the desired result.

Using the Córdoba and Córdoba inequality, an integration by parts and the previous decay property of $|\Lambda \phi|$, we obtain:

$$
\begin{aligned}
-\int w_{R} \phi \Lambda w_{R} d x \leq-\int \phi \Lambda\left(w_{R}^{2}\right) d x & =-\int \Lambda \phi w_{R}^{2} d x \\
& \leq \sum_{k \in \mathbb{Z}^{2}} \frac{1}{1+|k|^{3}} \int_{[-1,1]^{2}+k}\left|w_{R}\right|^{2} d x \\
& \lesssim \sup _{k \in \mathbb{Z}^{2}} \int_{[-1,1]^{2}+k}\left|w_{R}\right|^{2} d x \\
& \lesssim\left\|w_{R}\right\|_{H_{u l o c}^{s}}^{2} .
\end{aligned}
$$

For the third term we integrate by parts, and we use the fact that $u$ is divergence free to get

$$
\begin{aligned}
-\int \phi \Lambda^{s} w_{R} \nabla \cdot\left(u_{R} \Lambda^{s} w_{R}\right) d x= & \int \nabla \cdot\left(\phi \Lambda^{s} w_{R}\right) \cdot u_{R} \Lambda^{s} w_{R} d x \\
= & \int \phi \nabla\left(\Lambda^{s} w_{R}\right) \cdot u_{R} \Lambda^{s} w_{R} d x \\
& +\int \Lambda^{s} w_{R} \nabla \phi \cdot u_{R} \Lambda^{s} w_{R} d x \\
= & \int \phi \Lambda^{s} w_{R} \nabla \cdot\left(u_{R} \Lambda^{s} w_{R}\right) d x+\int \theta_{R}^{2} u_{R} \cdot \nabla \phi d x .
\end{aligned}
$$

Then, lemma 18 allows us to get

$$
\begin{aligned}
-\int \phi \Lambda^{s} w_{R} \nabla \cdot\left(u \Lambda^{s} w_{R}\right) d x=\frac{1}{2} \int \theta_{R}^{2} u_{R} \cdot \nabla \phi d x & \lesssim\left\|\theta_{R}\right\|_{L^{\infty}\left(\mathbb{R}^{2}\right)}\|\nabla \phi\|_{L^{\infty}}\left\|\theta_{R}\right\|_{L_{u l o c}^{2}\left(\mathbb{R}^{2}\right)}\left\|u_{R}\right\|_{L_{u l o c}^{2}\left(\mathbb{R}^{2}\right)} \\
& \lesssim\left\|w_{R}\right\|_{H_{u l o c}^{s}}^{2}\left(\mathbb{R}^{2}\right)
\end{aligned}
$$


Remark 20. Concerning this third term and more precisely the integration by parts, without regularization, we cannot integrate by parts since $\nabla \cdot\left(\phi \Lambda^{s} w_{R}\right) \in L^{2} \dot{H}^{-1 / 2}$ we must have $u_{R} \Lambda^{s} w_{R} \in$ $L^{2} \dot{H}^{1 / 2}$ which is done only after regularization.

Let us now estimate the last term; we use Lemma 4 of Córdoba and Córdoba applied to $\theta_{R}$, namely

$$
-\Lambda^{s} w_{R} \Lambda^{s+1} w_{R}=-\theta_{R} \Lambda \theta_{R} \leq-\frac{1}{2} \Lambda\left(\theta_{R}^{2}\right)
$$

We obtain

$$
\begin{aligned}
-\int \phi \Lambda^{s} w_{R} \Lambda^{s+1} w_{R} d x \leq-\int \phi \Lambda\left(\theta_{R}^{2}\right) d x & =-\int \Lambda \phi \theta_{R}^{2} d x \\
& \leq \sum_{k \in \mathbb{Z}^{2}} \frac{1}{1+|k|^{3}} \int_{k+[-1,1]^{2}}\left|\theta_{R}\right|^{2} d x \\
& \lesssim\left\|\theta_{R}\right\|_{L_{u l o c}^{2}}^{2} \\
& \lesssim\left\|w_{R}\right\|_{H_{u l o c}^{s}}^{2}\left(\mathbb{R}^{2}\right)
\end{aligned}
$$

Thus, we get the following estimate

$$
-\int \phi \Lambda^{s} w_{R} \Lambda^{s+1} w_{R} d x \leq C\left\|w_{R}\right\|_{H_{u l o c}^{s}}^{2}\left(\mathbb{R}^{2}\right) .
$$

All these estimates lead to

$$
\partial_{t} A_{\phi}\left(w_{R}\right) \leq C\left\|w_{R}\right\|_{H_{u l o c}^{s}}^{2}\left(\mathbb{R}^{2}\right)
$$

Integrating (5) in time $s \in[0, t]$ yields

$$
A_{\phi}\left(w_{R}(x, t)\right) \leq A_{\phi}\left(w_{0, R}(x)\right)+C \int_{0}^{t}\left\|w_{R}\right\|_{H_{u l o c}^{s}\left(\mathbb{R}^{2}\right)}^{2} d s .
$$

We now take the supremum over $\phi \in B$ to get

$$
\begin{aligned}
\left\|w_{R}(x, t)\right\|_{H_{u l o c}^{s}}^{2}\left(\mathbb{R}^{2}\right) & \leq\left\|w_{0, R}(x)\right\|_{H_{u l o c}^{s}\left(\mathbb{R}^{2}\right)}^{2}+C \int_{0}^{t}\left\|w_{R}(x, s)\right\|_{H_{u l o c}^{s}\left(\mathbb{R}^{2}\right)}^{2} d s \\
& \leq \sup _{R>1}\left\|w_{0, R}(x)\right\|_{H_{u l o c}^{s}\left(\mathbb{R}^{2}\right)}^{2}+C \int_{0}^{t}\left\|w_{R}(x, s)\right\|_{H_{u l o c}^{s}\left(\mathbb{R}^{2}\right)}^{2} d s .
\end{aligned}
$$

By the Gronwall inequality we conclude that

$$
\left\|w_{R}(x, t)\right\|_{L^{\infty}\left([0, T], H_{u l o c}^{s}\left(\mathbb{R}^{2}\right)\right)}^{2} \leq \sup _{R>1}\left\|w_{0, R}(x)\right\|_{H_{u l o c}^{s}\left(\mathbb{R}^{2}\right)}^{2} e^{C t} .
$$

Thus $(x, t) \mapsto w_{R}(x, t)$ is globally bounded in the space $L^{\infty}\left([0, T], \dot{H}_{u l o c}^{s}\left(\mathbb{R}^{2}\right)\right)$. We also need to have a uniform bound for $(x, t) \mapsto w_{R}(x, t)$ in the space $\left(L^{2} \dot{H}^{s+1 / 2}\right)_{u l o c}$. In other words, we would like to obtain a control of the norm

$$
\left\|w_{R}\right\|_{\left(L^{2} \dot{H}^{s+1 / 2}\right)_{u l o c}}^{2}=\left\|\theta_{R}\right\|_{\left(L^{2} \dot{H}^{1 / 2}\right)_{u l o c}}^{2}=\sup _{\phi \in B} \int_{0}^{T} \int \phi\left|\Lambda^{1 / 2} \theta_{R}\right|^{2} d x d s .
$$

We have seen that (see equality (4))

$$
\partial_{t} A_{\phi}\left(w_{R}\right)+\int \phi \theta_{R} \Lambda \theta_{R} d x \leq C\left\|w_{R}\right\|_{H_{u l o c}^{s}}^{2}
$$

Integrating in time $s \in[0, T]$ leads to

$$
A_{\phi}\left(w_{R}(x, T)\right)+\int_{0}^{T} \int \phi \theta_{R} \Lambda \theta_{R} d x d s \leq A_{\phi}\left(w_{0, R}(x)\right)+C \int_{0}^{T}\left\|w_{R}\right\|_{H_{u l o c}^{s}}^{2} d s .
$$


The last term in the left-hand side can be rewritten as

$$
\int_{0}^{T} \int \phi \theta_{R} \Lambda \theta_{R} d x d s=\int_{0}^{T} \int \Lambda^{1 / 2} \theta_{R}\left[\Lambda^{1 / 2}, \phi\right] \theta_{R} d x d s+\int_{0}^{T} \int \phi\left|\Lambda^{1 / 2} \theta_{R}\right|^{2} d x d s,
$$

therefore, the inequality (7) becomes

$$
\begin{aligned}
A_{\phi}\left(w_{R}(x, T)\right)+\int_{0}^{T} \int \phi\left|\Lambda^{1 / 2} \theta_{R}\right|^{2} d x d s \lesssim & A_{\phi}\left(w_{0, R}(x)\right)+\int_{0}^{T} C\left\|w_{R, \epsilon}\right\|_{H_{\text {uloc }}^{s}}^{2} d s \\
& +\left|\int_{0}^{T} \int \Lambda^{1 / 2} \theta_{R}\left[\Lambda^{1 / 2}, \phi\right] \theta_{R} d x d s\right|
\end{aligned}
$$

Then, we split the last integral in the right hand side as follows:

$$
\begin{aligned}
\int_{0}^{T} \int \Lambda^{1 / 2} \theta_{R}\left[\Lambda^{1 / 2}, \phi\right] \theta_{R} d x d s & =\int_{0}^{T} \int \psi \Lambda^{1 / 2} \theta_{R}\left[\Lambda^{1 / 2}, \phi\right] \theta_{R} d x d s \\
& +\int_{0}^{T} \int(1-\psi) \Lambda^{1 / 2} \theta_{R}\left[\Lambda^{1 / 2}, \phi\right] \theta_{R} d x d s . \\
& =(1)+(2) .
\end{aligned}
$$

For the first term, by Hölder and Young inequalities we have, for all $\nu>0$ :

$$
\begin{aligned}
\left|\int_{0}^{T} \int \psi \Lambda^{1 / 2} \theta_{R}\left[\Lambda^{1 / 2}, \phi\right] \theta_{R} d x d s\right| & \leq \int_{0}^{T}\left\|\psi \Lambda^{1 / 2} \theta_{R}\right\|_{L^{2}}\left\|\theta_{R}\right\|_{L_{u l o c}^{2}} d s \\
& \leq \frac{\nu}{2} \int_{0}^{T}\left\|\psi \Lambda^{1 / 2} \theta_{R}\right\|_{L^{2}}^{2} d s+\frac{1}{2 \nu} \int_{0}^{T}\left\|\theta_{R}\right\|_{L_{u l o c}^{2}}^{2} d s .
\end{aligned}
$$

For the second term, we also use Hölder and Young inequalities to obtain that, for all $\eta>0$, we have:

$$
\begin{aligned}
\int_{0}^{T} \int(1-\psi) \Lambda^{1 / 2} \theta_{R}\left[\Lambda^{1 / 2}, \phi\right] \theta_{R} d x d s & =\int_{0}^{T} \int(1-\psi) \Lambda^{1 / 2} \theta_{R} \Lambda^{1 / 2}\left(\phi \theta_{R}\right) d x d s \\
& =\int_{0}^{T} \int \Lambda^{1 / 2}\left((1-\psi) \Lambda^{1 / 2} \theta_{R}\right) \phi \theta_{R} d x d s \\
& \leq \sum_{|k|>5} \frac{1}{|k|^{5 / 2}} \int_{0}^{T} \int \phi_{k}\left|\Lambda^{1 / 2} \theta_{R}\right|\left|\phi \theta_{R}\right| d x d s \\
& \leq \frac{\eta}{2} \sum_{|k|>5} \frac{1}{|k|^{5 / 2}} \int_{0}^{T} \int \phi_{k}\left|\Lambda^{1 / 2} \theta_{R}\right|^{2} d x d s \\
& +\frac{1}{2 \eta} \int_{0}^{T}\left\|\theta_{R}\right\|_{L_{\text {uloc }}^{2}}^{2} d s,
\end{aligned}
$$

where we have written that $1-\psi=\sum_{|k|>5} \phi_{k}$. Then, we obtain the following inequality

$$
\begin{aligned}
& A_{\phi}\left(w_{R}(x, T)\right)+\int_{0}^{T} \int \phi\left|\Lambda^{s+1 / 2} w_{R}\right|^{2} d x d s \lesssim A_{\phi}\left(w_{0, R}(x)\right) \\
& +\left(\frac{1}{2 \eta}+\frac{1}{2 \nu}\right) \int_{0}^{T}\left\|\theta_{R}\right\|_{L_{u l o c}^{2}}^{2} d s+\left(\frac{\eta}{2}+\frac{\nu}{2}\right) \sup _{\psi \in B_{\psi}} \int_{0}^{T} \int \psi\left|\Lambda^{1 / 2} \theta_{R}\right|^{2} d x d s .
\end{aligned}
$$

We take the supremum over all $\phi \in B_{\phi}$ and then we choose $\nu$ and $\eta$ small enough so that the norm $\left\|\Lambda^{1 / 2} \theta_{R}\right\|_{\left(L^{2} L^{2}\right)_{u l o c}}^{2}$ appearing in the right hand side is absorbed by that of the left. We obtain

$$
\left\|w_{R}\right\|_{H_{u l o c}^{s}}^{2}+\left\|\Lambda^{1 / 2} \theta_{R}\right\|_{\left(L^{2} L^{2}\right)_{u l o c}}^{2} \lesssim\left\|w_{0, R}\right\|_{H_{u l o c}^{s}}^{2}+\int_{0}^{T}\left\|w_{R}\right\|_{H_{u l o c}^{s}}^{2} d s .
$$


By Gronwall's lemma, we recover the following control, for all $T>0$

$$
\left\|w_{R}\right\|_{L^{\infty}\left([0, T], H_{u l o c}^{s}\right)}^{2} \leq\left\|w_{0, R}\right\|_{H_{u l o c}^{s}}^{2} e^{C T} .
$$

This readily gives us the desired $\left(L^{2} \dot{H}^{s+1 / 2}\right)$ uloc bound.

The final step is to show that the solutions $w_{R, \epsilon}$ converge weakly to the solutions $w$, and that $w$ are weak solutions of $(S Q G)$.

\section{Passage to the limit $(R \rightarrow \infty, \epsilon \rightarrow 0)$}

In this section, we show that the solutions of the truncated $(S Q G)_{R, \epsilon}$ equation tend to the solutions of the classical $(S Q G)$ equation, and that the limit has the expected regularity.

Let $\eta:(t, x) \mapsto \eta(t, x) \in \mathcal{D}\left([0, T] \times \mathbb{R}^{2}\right)$ be a positive test function. Clearly, we have

$$
\left\langle\partial_{t} \theta_{R, \epsilon}, \eta\right\rangle=-\left\langle\theta_{R, \epsilon}, \partial_{t} \eta\right\rangle \underset{R \rightarrow+\infty}{\longrightarrow}-\left\langle\theta_{\epsilon}, \partial_{t} \eta\right\rangle
$$

Thus,

$$
\partial_{t} w_{R, \epsilon} \underset{R \rightarrow+\infty, \epsilon \rightarrow 0}{\longrightarrow} \partial_{t} w \text { in } \mathcal{D}^{\prime}\left([0, \mathrm{~T}] \times \mathbb{R}^{2}\right) .
$$

Now, let us focus on the convergence of the non linear term. As usual, weak convergence is not enough to pass to the limit in the non linear term; we need a strong convergence of $\theta_{R, \epsilon}$ which is given by the Rellich compactness theorem.

Since $\theta_{R, \epsilon}$ is uniformly bounded with respect to $R$ and $\epsilon$ in $\left(L^{2} \dot{H}^{1 / 2}\right)_{l o c}$ and $u_{R, \epsilon}$ is uniformly bounded in the space $\left(L^{2} L^{2}\right)_{l o c}$. Therefore, by the Sobolev embedding, the product $\theta_{R, \epsilon} u_{R, \epsilon}$ is uniformly bounded in the space $\left(L^{2} L^{4}\right)_{l o c} \cap\left(L^{2} L^{2}\right)_{l o c} \subset\left(L^{2} L^{4 / 3}\right)_{l o c} \subset\left(L^{2} H^{-1 / 2}\right)_{l o c}$, thus $\nabla . \theta_{R, \epsilon} u_{R, \epsilon}$ is uniformly bounded in the space $\left(L^{2} \dot{H}^{-3 / 2}\right)_{l o c}$. Moreover, since $\theta_{R, \epsilon}$ is uniformly bounded in $\left(L^{2} \dot{H}^{1 / 2}\right)_{l o c}$ then $\Lambda \theta_{R, \epsilon}$ is bounded in $\left(L^{2} \dot{H}^{-1 / 2}\right)_{l o c}$. We infer that $\partial_{t} \theta_{R, \epsilon}$ is bounded in $\left(L^{2} \dot{H}^{-3 / 2}\right)_{l o c}$. By Rellich (see e.g [16], Lemma 21.5, p 218), we get the strong convergence

$$
\theta_{R, \epsilon} \underset{R \rightarrow+\infty, \epsilon \rightarrow 0}{\longrightarrow} \theta \text { in }\left(L^{2} L^{2}\right)_{l o c}
$$

Therefore $\nabla \cdot\left(u_{R, \epsilon} \theta_{R, \epsilon}\right)$ converges weakly (up to a subsequence) when $R \rightarrow+\infty, \epsilon \rightarrow 0$, toward $\nabla .(u \theta)$ in the space $\left(L^{2} H^{-3 / 2}\right)_{l o c}$ and thus also in $\mathcal{D}^{\prime}\left((0, T], \mathbb{R}^{2}\right)$. Moreover, $\Lambda \theta_{R, \epsilon}$ is uniformly bounded in $\left(L^{2} \dot{H}^{-1 / 2}\right)_{l o c}$, then $\Lambda \theta_{R, \epsilon}$ converges weakly (up to a subsequence) when $R \rightarrow+\infty, \epsilon \rightarrow 0$ toward $\Lambda w$ in $\left(L^{2} H^{-1 / 2}\right)_{\text {loc }}$ and thus in $\mathcal{D}^{\prime}\left((0, T], \mathbb{R}^{2}\right)$. We conclude that, for all $\eta$ in $\mathcal{D}\left([0, T] \times \mathbb{R}^{2}\right)$ we have

$$
\left\langle\eta(t, x), \partial_{t} w\right\rangle=\left\langle\left(\Lambda^{-s} \nabla\right) \cdot\left(\Lambda^{s} w\left(\mathcal{R}^{\perp} \Lambda^{s} w\right)\right)-\Lambda w, \eta\right\rangle
$$

The next step is to show that $\theta$ and $w$ have the expected regularity. Let us recall that the spaces $L_{\text {uloc }}^{p}($ with $p>1)$ and $H_{\text {uloc }}^{s}($ with $s \in \mathbb{R})$ are respectively the dual spaces of the following separable Banach spaces:

$$
\begin{aligned}
W L^{p^{\prime}}\left(\mathbb{R}^{2}\right) & =\left\{\theta \in L_{l o c}^{p^{\prime}}\left(\mathbb{R}^{2}\right), \sum_{k \in \mathbb{Z}^{2}}\|\theta\|_{L^{p^{\prime}}\left(k+[0,1]^{2}\right)}<\infty\right\} \\
W H^{-s}\left(\mathbb{R}^{2}\right) & =\left\{\theta \in H_{l o c}^{-s}\left(\mathbb{R}^{2}\right), \sum_{k \in \mathbb{Z}^{2}}\|\phi(x-k) \theta\|_{H^{-s}\left(\mathbb{R}^{2}\right.}<\infty\right\},
\end{aligned}
$$

where $1 / p+1 / p^{\prime}=1$.

By Rellich, we also get the weak convergence (up to a subsequence):

$$
\theta_{R, \epsilon} \underset{R \rightarrow+\infty, \epsilon \rightarrow 0}{\longrightarrow} \theta \text { weakly in }\left(L^{2} \dot{H}^{1 / 2}\right)_{l o c}
$$


Since we have obtained that $w_{R, \epsilon}$ is uniformly bounded in $\left(L^{\infty} H^{s}\right)_{u l o c}$ which is the dual space of the Banach space $L^{1} W H^{-s}$ we infer that $w \in \cap_{t<T}\left(L^{\infty}\left([0, t], H^{s}\right)\right)_{u l o c}$ and then $\theta \in$ $\cap_{t<T}\left(L^{\infty}\left([0, t], L^{2}\right)\right)_{u l o c}$.

From the uniform bounds, $w_{R, \epsilon} \in\left(L^{2} \dot{H}^{s+1 / 2}\right)_{u l o c}$ which is the dual space of the Banach space $L^{2} W \dot{H}^{-s-1 / 2}$ therefore we get that

$$
w \in \cap_{t<T}\left(\left(L^{2}\left([0, t], \dot{H}^{s+1 / 2}\right)\right)_{u l o c}\right.
$$

and then

$$
\theta \in \cap_{t<T}\left(L^{2}\left([0, t], \dot{H}^{1 / 2}\right)\right)_{u l o c}
$$

For the initial data we have the following convergence:

$$
\begin{aligned}
& \theta_{0, R, \epsilon} \underset{R \rightarrow+\infty, \epsilon \rightarrow 0}{\longrightarrow} \theta_{0} * \text {-weakly in } L^{\infty}, \\
& w_{0, R, \epsilon} \underset{R \rightarrow+\infty, \epsilon \rightarrow 0}{\longrightarrow} w_{0} * \text {-weakly in } H_{\text {uloc }}^{s}
\end{aligned}
$$

Let us show the first convergence. For all $\phi \in L^{1}$ we have:

$$
\left\langle\theta_{0, R, \epsilon}, \phi\right\rangle=\left\langle\theta_{0, R}, \phi * \rho_{\epsilon}\right\rangle \underset{\epsilon \rightarrow 0}{\longrightarrow}\left\langle\theta_{0, R}, \phi\right\rangle,
$$

and thanks to the uniform bounds previously obtained, namely

$$
\begin{aligned}
\left\langle\theta_{0, R}, \phi\right\rangle & =\left\langle\chi_{R} \theta_{0}, \phi\right\rangle+\left\langle\left[\Lambda^{s}, w_{0, R}\right] \chi_{R}, \phi\right\rangle \\
& \leq\left\langle\chi_{R} \theta_{0}, \phi\right\rangle+R^{-s}\|\nabla \chi\|_{L^{\infty}}\left\|w_{0}\right\|_{L^{\infty}}+2 R^{-s}\left\|\chi_{R}\right\|_{L^{\infty}}\left\|w_{0}\right\|_{L^{\infty}},
\end{aligned}
$$

we infer that

$$
\left\langle\theta_{0, R}, \phi\right\rangle \underset{R \rightarrow+\infty}{\longrightarrow}\left\langle\theta_{0}, \phi\right\rangle * \text {-weakly in } L^{\infty} .
$$

For the convergence (10), we have seen that $w_{0, R, \epsilon}$ is uniformly bounded in $H_{u l o c}^{s}$, therefore, since $H_{u l o c}^{s}$ is a dual space, we get the following convergence (up to a subsequence)

$$
w_{0, R, \epsilon} \underset{R \rightarrow+\infty, \epsilon \rightarrow 0}{\longrightarrow} w_{0} \quad *-\text { weakly in } H_{u l o c}^{s} .
$$

and we conclude that

$$
\theta_{0, R, \epsilon} \underset{R \rightarrow+\infty, \epsilon \rightarrow 0}{\longrightarrow} \theta_{0} \quad * \text {-weakly in } \Lambda^{s}\left(H_{u l o c}^{s}\right) .
$$

Passing to the weak limit in (6), we obtain

$$
\|w(x, t)\|_{L^{\infty} H_{u l o c}^{s}}^{2}\left(\mathbb{R}^{2}\right) \lesssim e^{C t}
$$

Thus, for $1 / 2<s<1$, the solution does not blow up in finite time so we have global existence of weak solutions when $\theta_{0} \in \Lambda^{s}\left(H_{\text {uloc }}^{s}\left(\mathbb{R}^{2}\right)\right) \cap L^{\infty}\left(\mathbb{R}^{2}\right)$.

Concluding remarks. Actually, the energy inequality obtained in Sect.3.4 can be improved and we can show that in the range $1 / 4 \leq s \leq 1 / 2$ we also have global existence this is done in [15]. The case $0<s<1 / 4$ is little more technical because of the first term among the fourth terms studied in Sect.3.4, namely

$$
-\int \nabla \Lambda^{-s}\left(w_{R} \phi\right) u_{R} \theta_{R} d x
$$

This term is not easy to handle because of the lack of regularity. Nevertheless, in that case, we can show that we have local existence of weak solutions for large initial data in $\Lambda^{s}\left(H_{u l o c}^{s}\left(\mathbb{R}^{2}\right)\right)$, this is also done in the forthcoming work [15].

Let us note that the only role of the critical dissipation term $\Lambda \theta$ is to use the result of Abidi and Hmidi [1]. This latter ensures us the local boundedness of the Riesz transform in $L_{t}^{\infty} L^{\infty}$ which is a crucial point in our proof.

Finally, let us point out that the uniqueness of those global solutions is open. As it is well known, this kind of proof based on energy and compactness does not provide uniqueness. 
Acknowledgment: I thank my advisor Prof. Pierre-Gilles Lemarié-Rieusset who brought this subject to my attention for his great help and advices.

\section{References}

[1] Abidi H., Hmidi T.: On the global well-posedness of the critical quasi-geostrophic equation, SIAM J. Math. Anal. 40 (2008), no. 1, 167-185.

[2] Basson A.: Homogeneous Statistical Solutions and Local Energy Inequality for 3D NavierStokes Equations, Comm. Math. Phys. 266 (2006), p. 17-35

[3] Bahouri H., Chemin J.-Y., Danchin, R.: Fourier Analysis and Nonlinear Partial Differential Equations, 343p, Springer Verlag, 2011.

[4] Caffarelli, L., Vasseur, A.: Drift diffusion equations with fractional diffusion and the quasigeostrophic equation, Annals of Math (2010) 171, no. 3. 1903-1930.

[5] Cannone, M.: Ondelettes, paraproduits et Navier-Stokes, Diderot éditeur, Paris, 1995.

[6] Chae, D., Lee, J.: Global well-posedness in the super-critical dissipative quasi-geostrophic equations, Comm. Math. Phys. 233(2003), pp. 297-311.

[7] Constantin, P., Córdoba, D., Wu, J.: On the critical dissipative quasi-geostrophic equation, Indiana Univ. Math. J, 50(2001), pp. 97-107.

[8] Constantin, P., Majda, A.J., Tabak, E.: Formation of strong fronts in the 2-D quasigeostrophic thermal active scalar, Nonlinearity(1994), 7, pp. 1495-1533.

[9] Constantin, P., Wu, J.: Behavior of solutions of 2D quasi-geostrophic equations. SIAM J. Math. Anal. 30, 937-948 (1999).

[10] Córdoba, A., Córdoba, D.: A maximum principle applied to quasi-geostrophic equations, Comm. Math. Phys. 249(2004), pp. 511-528.

[11] Córdoba, D.: Nonexistence of simple hyperbolic blow-up for the quasi-geostrophic equation, Annals of Math, 148(1998), no. 3, pp. 1135-1152.

[12] Ju, N.: Existence and uniqueness of the solution to the dissipative 2D quasi-geostrophic equations in the Sobolev space. Comm. Math. Phys. 251(2004), no. 2, pp. 365-376.

[13] Kiselev, A., Nazarov, F., Volberg, A.: Global well-posedness for the critical $2 d$ dissipative quasi-geostrophic equation, Invent. Math (2007). 167, no. 3, 445-453.

[14] Marchand, F.: Existence and regularity of weak solutions to the quasi-geostrophic equations in the spaces $L^{p}$ or $\dot{H}^{-1 / 2}$, Comm. Math. Phys. 277, 45-67 (2008).

[15] Lazar, O.: Global and Local existence for the dissipative critical SQG equation with small oscillations, preprint.

[16] Lemarié-Rieusset, P. G.: Recent developments in the Navier-Stokes problem. Chapman \& Hall/CRC, (2002).

[17] Lemarié-Rieusset, P. G.: The Navier-Stokes equations in the critical Morrey-Campanato space, Revista Mat. Ibero-amer. 23, pp. 897-930 (2007).

[18] Resnick, S.:Dynamical problems in nonlinear advective partial differential equations, Ph.D. Thesis, University of Chicago, (1995).

[19] Vishik, M.I., Fursikov, A.V.: Solutions statistiques homogènes des systèmes différentiels paraboliques et du système de Navier-Stokes, Ann. Scuola Norm. Sup. Pisa, série IV (1977), pp. 531-576. 
[20] Vishik, M.I., Fursikov, A.V.: Translationally homogeneous statistical solutions and individual solutions with infinite energy of a system of Navier-Stokes equations, Siberian Math. Journal 19 (1978), pp. 710-729.

[21] Wu, J: Global solutions of the 2D dissipative quasi-geostrophic equation in Besov spaces. SIAM J. Math. Anal, 36(2005), pp. 1014-1030. 